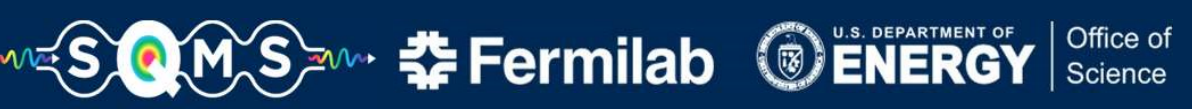

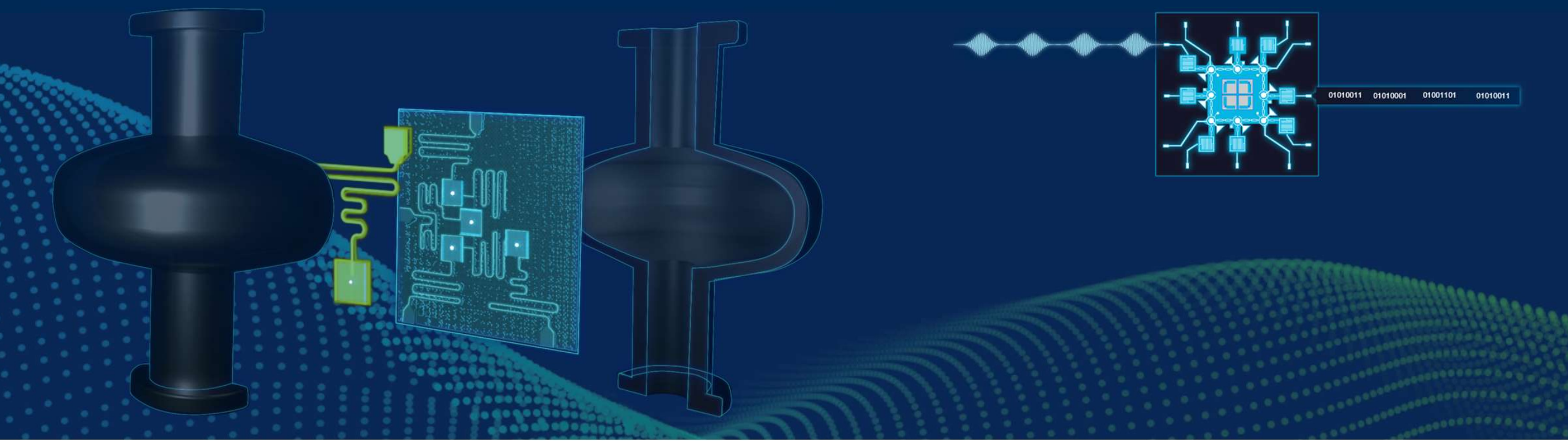

\title{
Cryogenic infrastructure for quantum computing
}

Matthew Hollister

Fermi National Accelerator Laboratory

International Cryogenic Materials Conference 2021

M1Or1B-04

FERMILAB-SLIDES-21-053-TD 


\section{Presentation outline}

- The needs of quantum computing

- Larger cryogenic platforms

- Larger cryogenic facilities

- Concluding remarks 


\section{Cryogenics and quantum computing}

- Many flavors of quantum processor are being explored, not all of them cryogenic (yet).

- The designs that have seen the most development are generally based on superconducting RF resonators - for example "transmons", but there are a number of other architectures.

- The common feature of these devices are that they either rely on a cryogenic phenomenon such as superconductivity, or they benefit from a low thermal noise environment. 


\section{Ion trap computers}

- Another very promising approach is a cryogenic ion trap.

- The ion trap itself is an electromagnet device, but issues relating to thermal noise affecting the coherence of the trapped ions can be addressed by combining the trap with a cryogenic system at $\sim 10 \mathrm{~K}$

Cryogenic ion trap system at the University of Sussex. System combines a 4-K cryocooler with an ion trap (Image: University of Sussex)

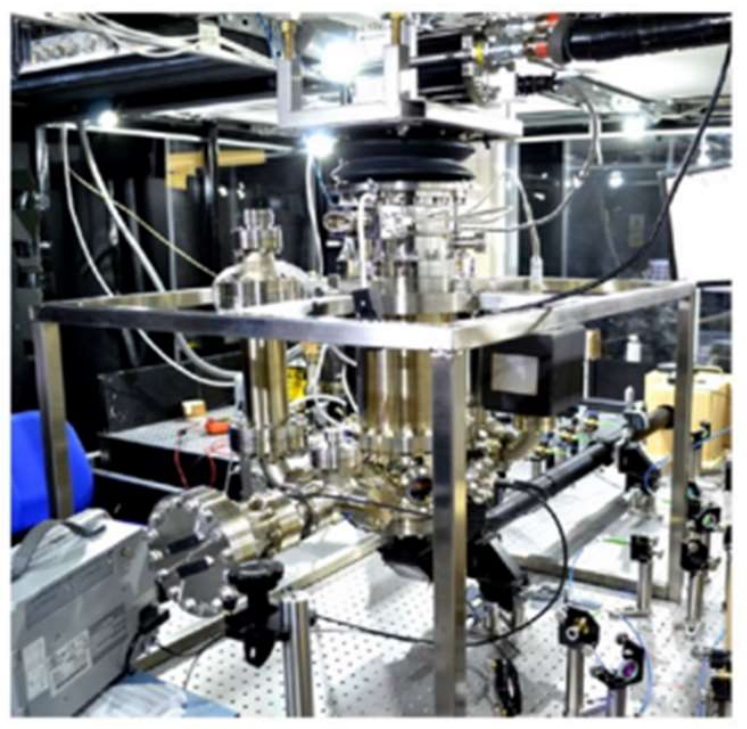




\section{Superconducting qubits}

- Superconducting RF and similar qubits are intimately connected to dilution refrigerators - the continuous low temperature cooling is ideal for stable cryogenic devices.

- Current state of the art processors have $\sim 100$ qubits.

- Larger quantum processors need bigger cryogenic platforms with more cooling capacity.

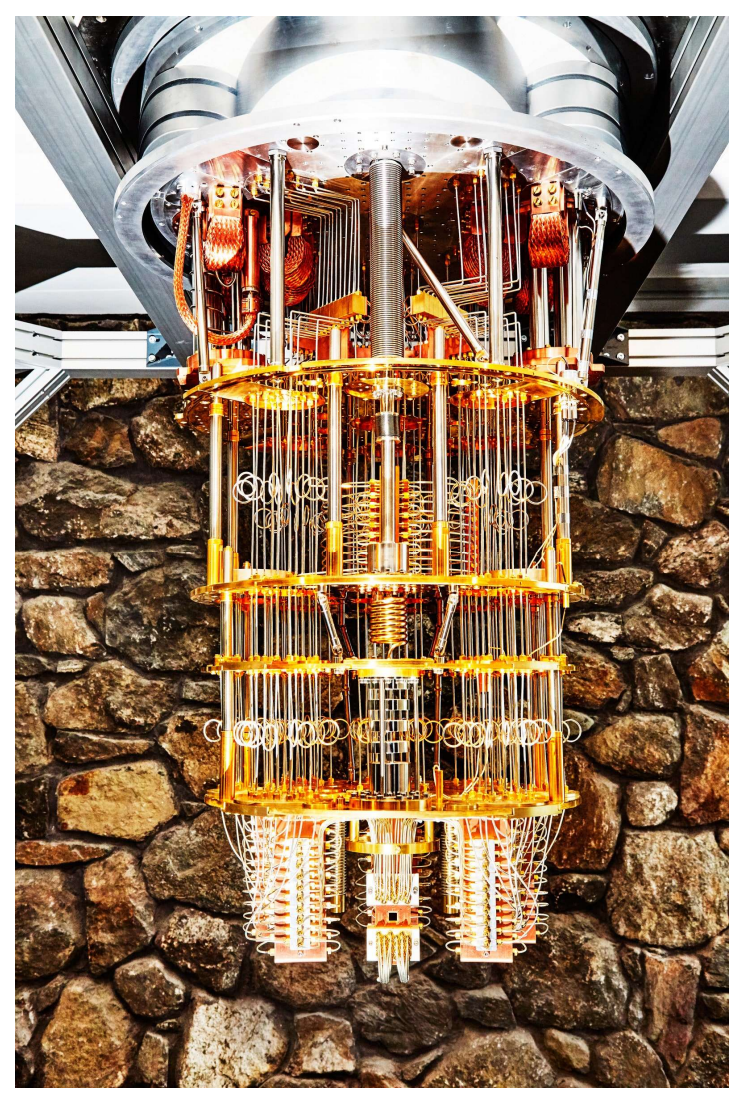

Image: IBM / Amy Lombard

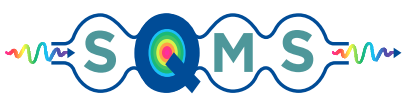




\section{Larger dilution cryostats}

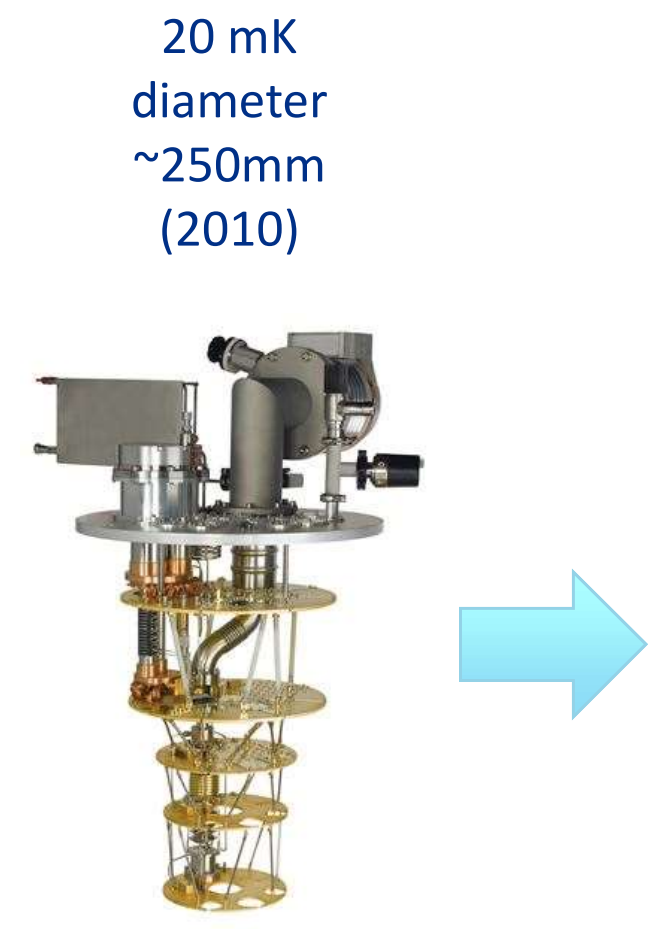

Image: Oxford Instruments

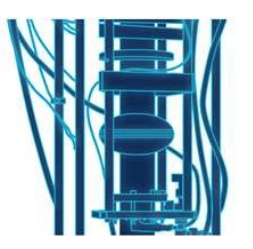

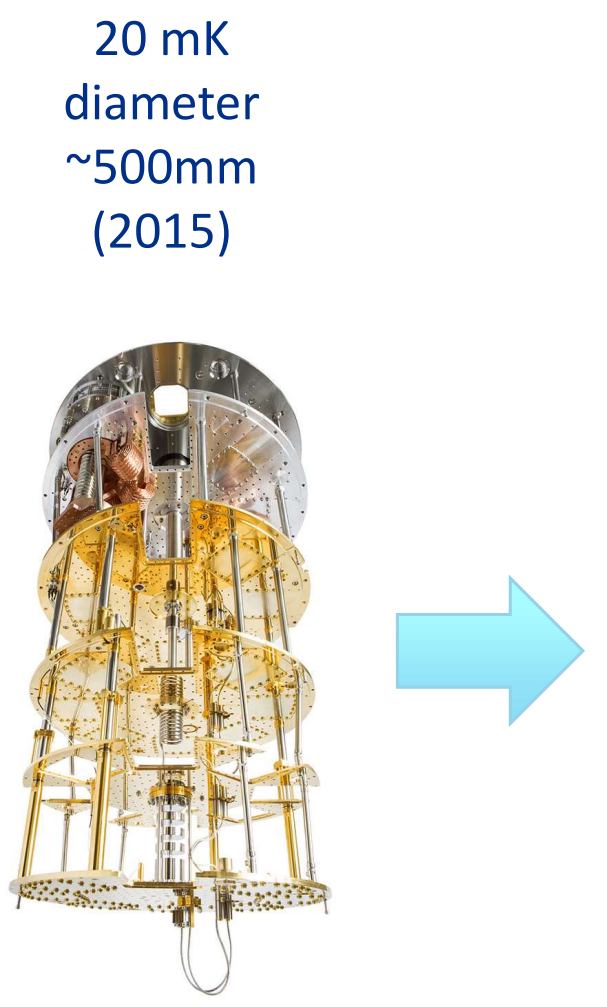

Image: BlueFors
$20 \mathrm{mK}$

diameter

$\sim 1000 \mathrm{~mm}$

(2021)

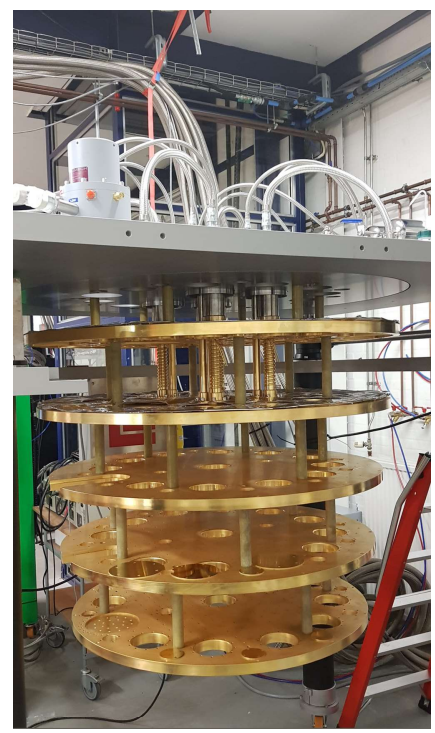

Image: Leiden Cryogenics

\section{???}

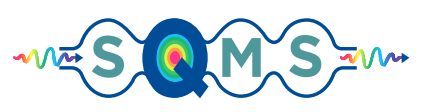

Cryogenic infrastructure for quantum computing

SUPERCONDUCTING QUANTUM MATERIALS \& SYSTEMS CENTER 


\section{Some issues with scaling up}

- It is not feasible to arbitrarily scale up a mK cryogenic platform due to the nature of the dilution process.

- Capacity of mechanical coolers used in fridge platforms is also a limiting factor in larger systems.

- Before we explore these two factors, we will very briefly discuss the design of a modern dilution refrigerator. 


\section{A quick tour of a dilution refrigerator}

\section{Image: Oxford Instruments}

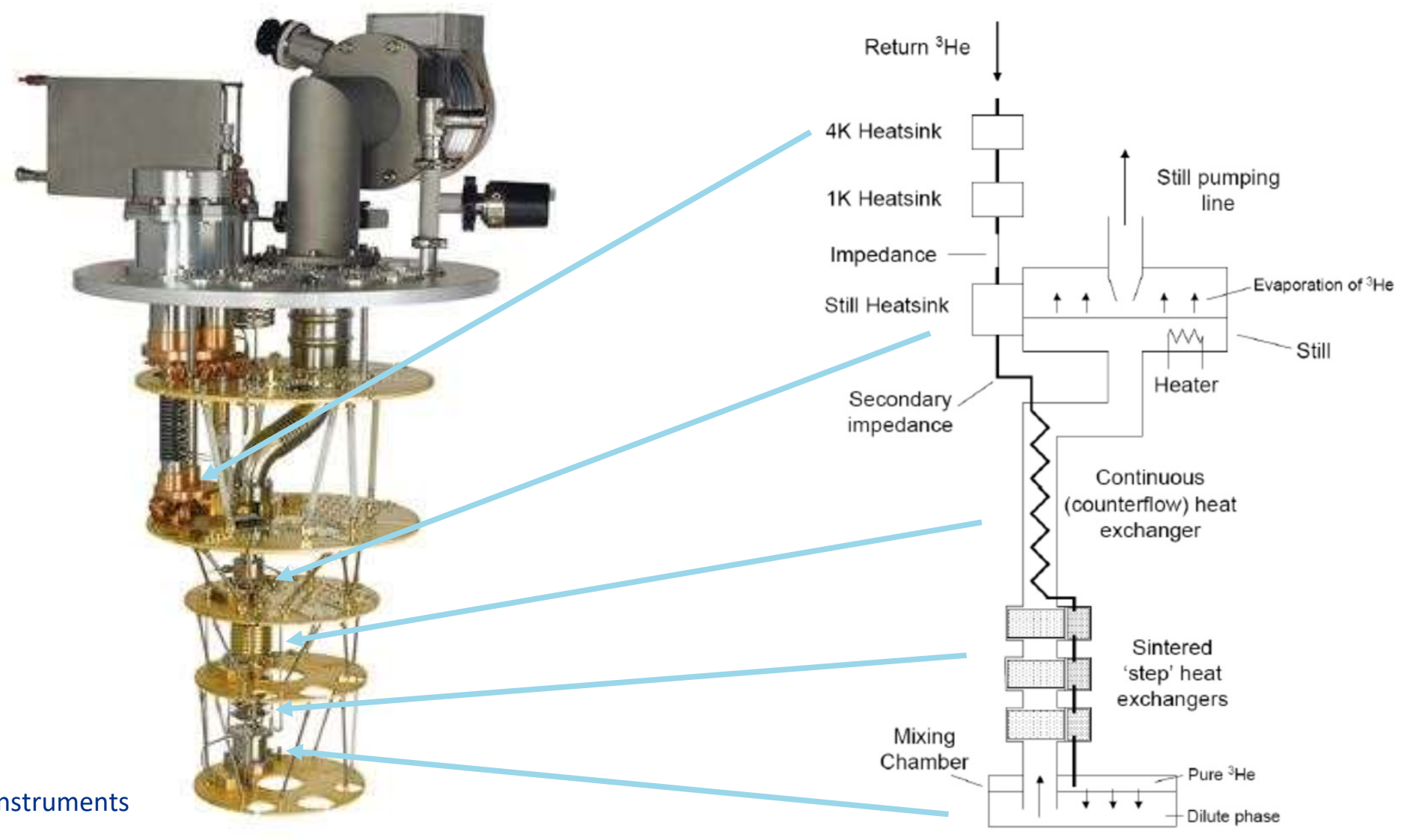




\section{Circulation and heat exchange}

- The cooling power of a dilution refrigerator comes from the heat of mixing of pure $3 \mathrm{He}$ into a mixture of $3 \mathrm{He}$ and $4 \mathrm{He}$.

- Cooling power of the dilution process is proportional to the 3 He circulation rate, usually quoted as

$$
Q_{M C}=84 n_{3} T_{M C}^{2}
$$

where $Q_{M C}$ is the cooling capacity, $n_{3}$ is the $3 \mathrm{He}$ circulation rate, and $T_{M C}$ is the mixing chamber temperature 


\section{Circulation and heat exchange (2)}

- The quoted equation is idealized, however. The full derivation actually yields a relationship that includes $T_{N}$, the temperature of the $3 \mathrm{He}$ as it enters the mixing chamber:

$$
Q_{M C}=n_{3}\left(96 T_{M C}^{2}-12 T_{N}^{2}\right)
$$

- From this, it can be seen that, for practical purposes, the cooling power of the fridge is really a function of how effective the heat exchangers are. This applies to the heat exchangers higher up the system as much as down at the cold end of the process. 


\section{Precooling and experimental loads}

- In addition to the cold end of the dilution refrigerator, we must also consider the cooling of the $3 \mathrm{He}$ as it flows from room temperature, and the demands of experimental wiring.

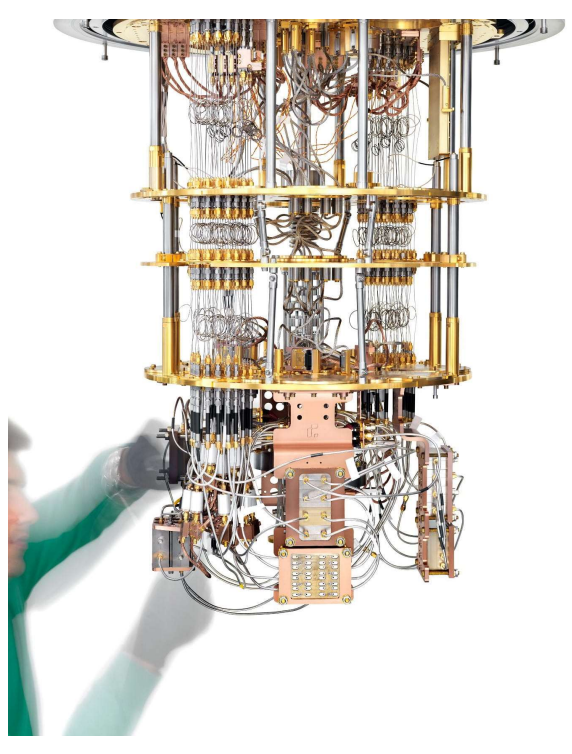

A typical quantum computer setup Image: Rigetti Computing / Justin Fantl
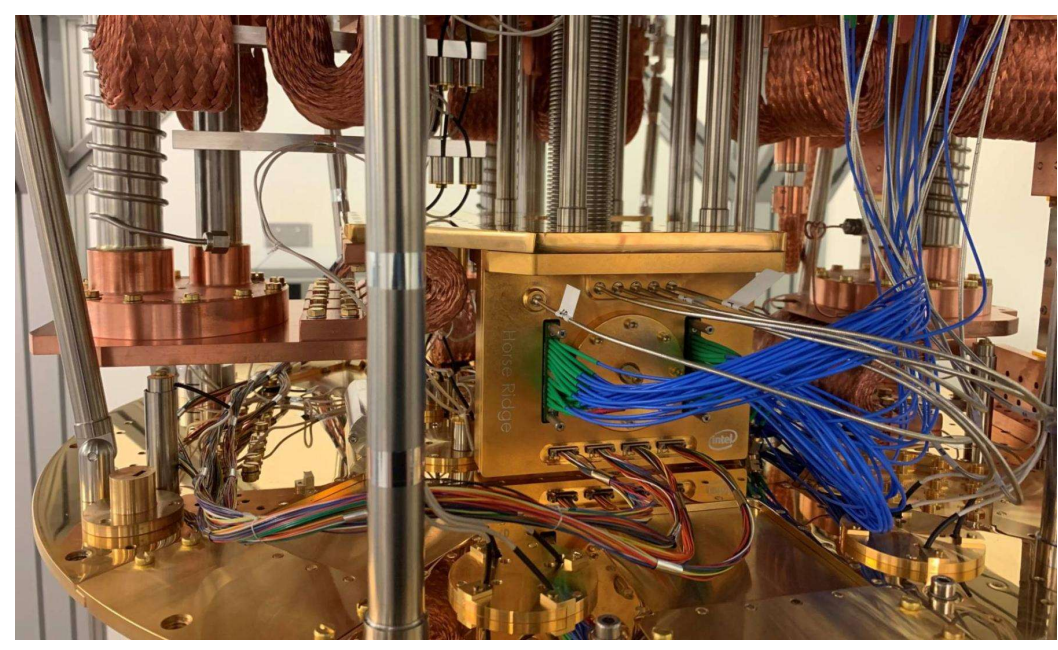

Cryogenic control processor Image: Intel 


\title{
Quantum networking
}

- Schemes to network quantum computers typically include some method of transduction to convert microwave photons to optical while maintaining coherence.

- Advantageous if the transduction is cryogenic to minimize noise sources.

- Photons can then be transmitted via optical link to another quantum processor.

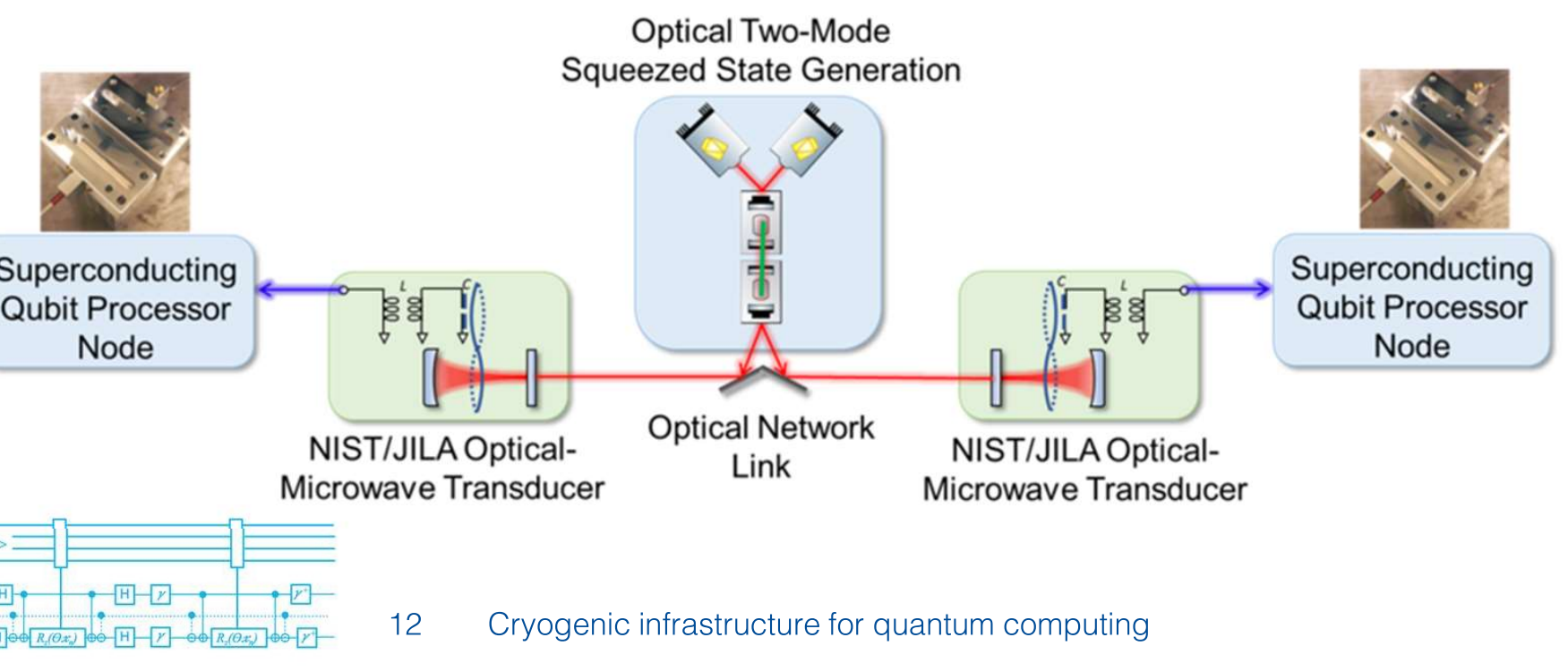

\author{
Quantum \\ networking \\ demonstration \\ (image: NIST / \\ Tasshi Dennis)
}

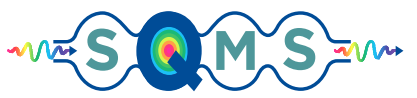

SUPERCONDUCTING QUANTUM MATERIALS \& SYSTEMS CENTER 


\section{Large platforms with an extra thermal stage}

- A cryostat with a high Helium-3 circulation and/or a large load at intermediate temperatures can be realized using a pumped 4He stage.

- Analogous to the "1-K Pot" on an older style dilution refrigerator.

- This configuration was described in the literature in 2009 (Hollister \& Woodcraft, Cryogenics) and demonstrated in 2015 (Uhlig, Cryogenics).

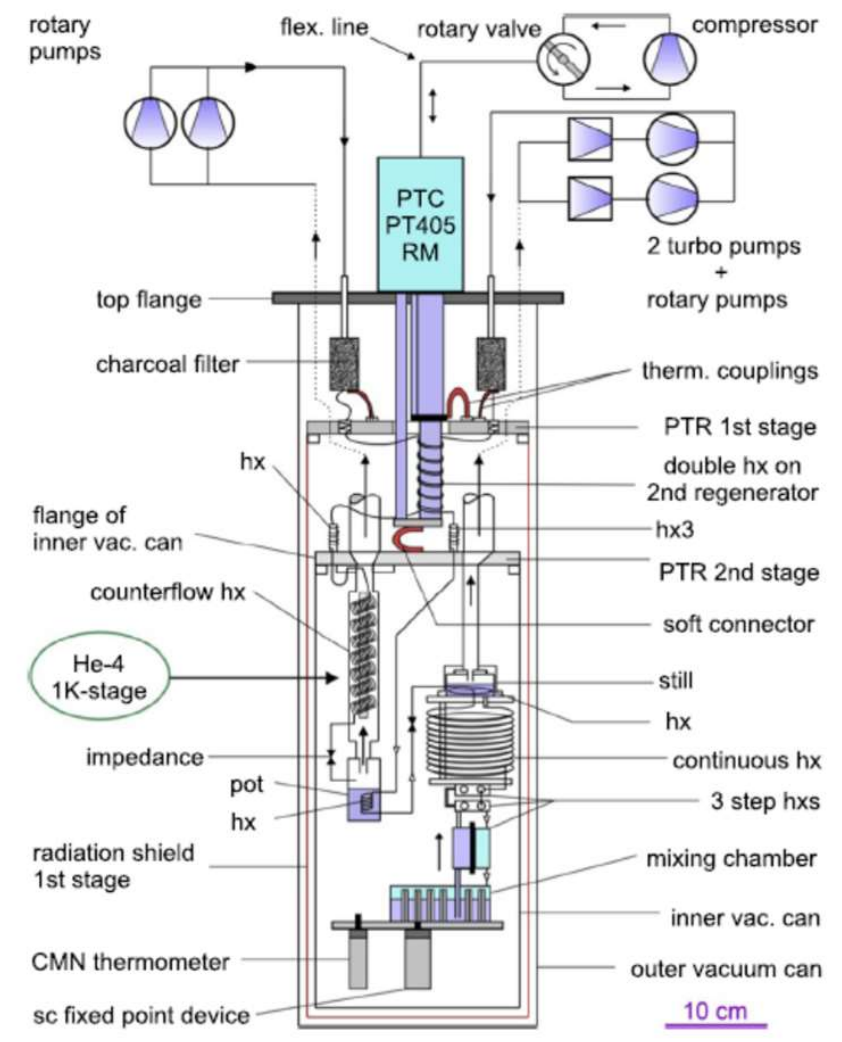

Dilution refrigerator with a $1-\mathrm{K}$ condenser (Image: K. Uhlig)

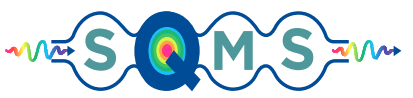

SUPERCONDUCTING QUANTUM MATERIALS \& SYSTEMS CENTER 


\section{Large platforms using liquid helium}

- Another possibility for the construction of a large platform is to utilize liquid or supercritical helium rather than cryocoolers.

- This is the approach taken in the design of the large mK platform under construction at Fermilab - see my talk later today (M1OraA03)

- The platform uses a 600W / $4.2 \mathrm{~g} / \mathrm{s}$ cryoplant

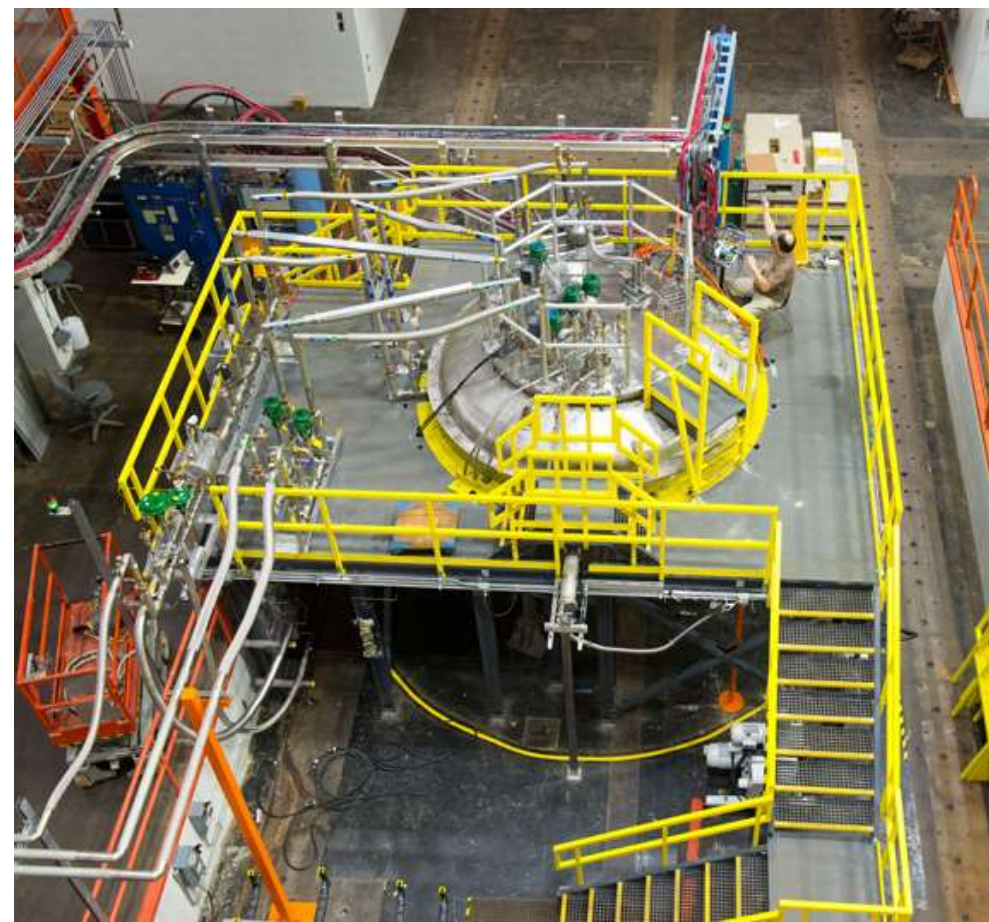

Exterior of the large $\mathrm{mK}$ platform at Fermilab Image: Fermilab 


\section{Larger cryogenic facilities - a concept for a "quantum data center"}

- The most likely scheme for the deployment of quantum computers will be cloud-based, with "data centers" of many quantum processors (perhaps networked) located together in a way analogous to supercomputing centers.

- This is already being done on a small scale by the bigger industry players such as IBM.

- This leads to some interesting infrastructure possibilities

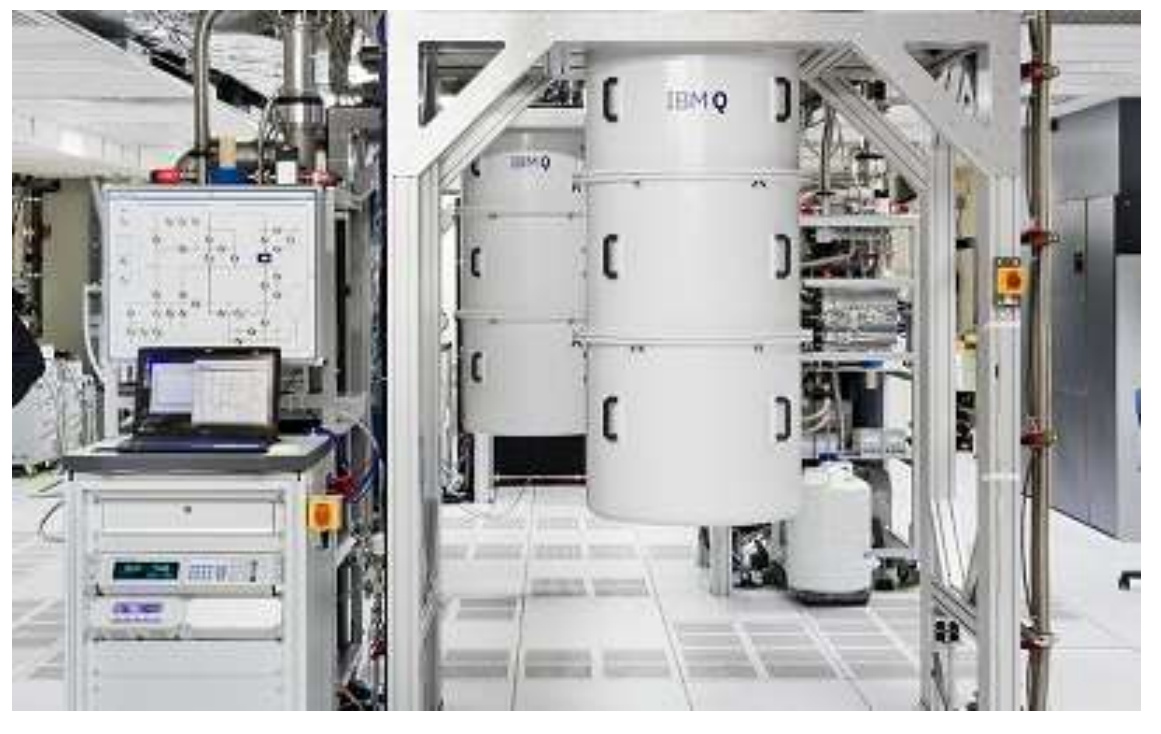

Image: IBM 


\section{Larger cryogenic facilities - a concept for a "quantum data center"}

- As discussed previously, the use of liquid helium rather than mechanical coolers makes the construction of large individual platforms possible but can also support multiple small cryostats from a central plant.

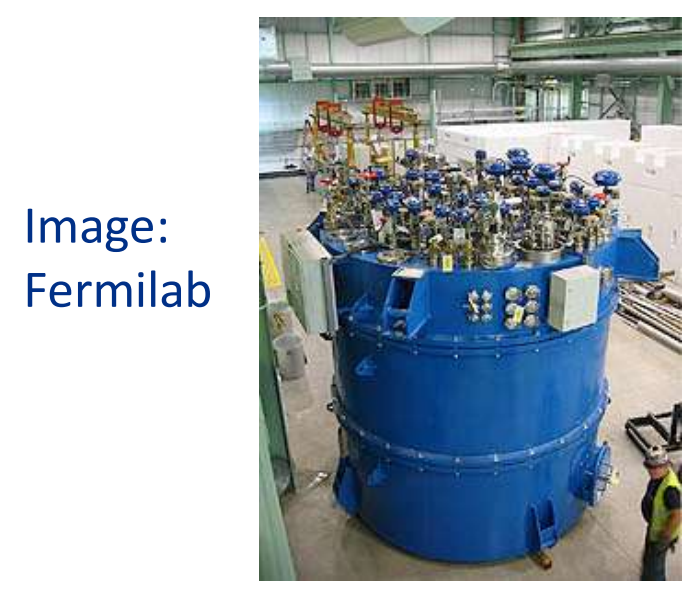

Central LHe plant
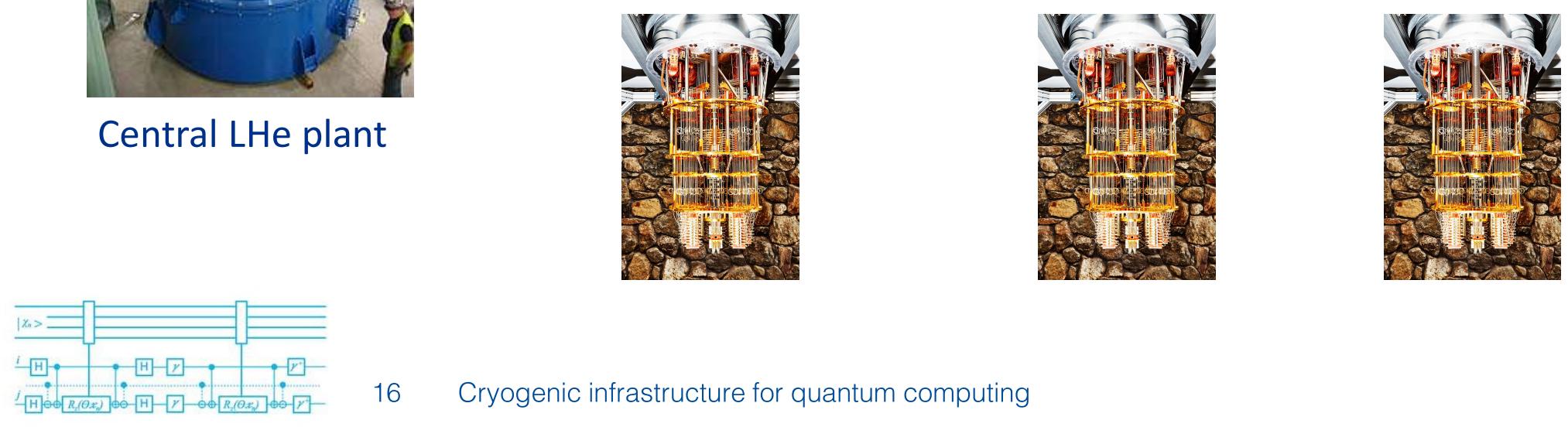


\section{Concluding remarks}

- Progress in the last decade on cryogenic technologies in the $\mathrm{mK}$ range has been extremely rapid, not doubt driven by commercial interests.

- Technology is still in infancy in many ways - the "scale up by brute force" approach has been successful so far, but this is not a sustainable path.

- There is a broad range of cryogenic expertise that has yet to be applied to the field of cryogenics for quantum.

- Likely to be some very exciting developments in the next five to ten years, both in the quantum computing field itself but also the cryogenic infrastructure. 


\section{Acknowledgement}

This manuscript has been authored by Fermi Research Alliance, LLC under Contract No. DE-AC02$07 \mathrm{CH} 11359$ with the U.S. Department of Energy, Office of Science, Office of High Energy Physics. 